\title{
Evaluation of Real-Time Catch and Effort Reporting in the U.S. Illex illecebrosus Fishery
}

\author{
Eric N. Powell and Allison J. Bonner \\ Haskin Shellfish Research Laboratory, Rutgers University \\ 6959 Miller Avenue, Port Norris, NJ 08349, USA \\ Roger Mann \\ Virginia Institute of Marine Sciences, College of William and Mary \\ Gloucester Point, VA 23062, USA \\ Sarah E. Banta \\ College of Oceanic and Atmospheric Sciences, Oregon State University \\ 104 Ocean Administration Bldg, Corvallis, OR 97331, USA
}

\begin{abstract}
The Illex illecebrosus fishery in the northwestern Atlantic Ocean is a trawl-based fishery. Illex illecebrosus normally lives less than one year, thus managing the fishery using a standard stock assessment approach is difficult. Real-time management is an attractive option. The purpose of this study was to investigate the validity and utility of data reported by commercial vessels for application in real-time management in the I. illecebrosus fishery. The assessment includes evaluating the frequency of erroneous reports, identifying the best measures of effort for catch per unit effort (CPUE) analysis, and examining the influence of vessel type and vessel behavior on subsequent analyses of trends in catch and effort.

Incomplete reports occurred $24 \%$ of the time in the commercial data. Misreported times and positions occurred less than $1 \%$ of the time. Searching time was not discriminated consistently. Searching times and distances were recovered from the more accurately-reported data on tow times and tow positions. Tow duration was more accurately estimated from Captains' reports than tow distance. True distances were underestimated by $10-20 \%$ using the position of net deployment and retrieval to measure tow distance because tows did not follow a straightline path. Effort could be estimated based on tow distance or tow duration. Duration was the most accurate effort term. Searches between tows did not improve subsequent catch. Similarly, overnight transits, arguably a type of searching behavior, did not improve the subsequent day's catch. Thus, vessels, on the average, would have improved trip economics by not searching. Because of the apparent absence of a positive effect of searching and the lower accuracy of searching time and searching distance in comparison to tow-based metrics, particularly tow duration, addition of searching time or searching distance to effort did not materially improve the measurement of CPUE.

RSW (refrigerated seawater) trawlers search much less often than freezer trawlers. As a consequence of lower catch and longer duration tows, the CPUE for RSW trawlers is distinctly lower than for freezer trawlers. Catch per tow was higher by more than a factor of two for larger vessels. As a consequence of the higher catch and longer tows, CPUE was significantly higher also for the larger vessels. Managing the I. illecebrosus fishery using an industry-based reporting scheme would require information from freezer and RSW trawlers and specified vessel size categories.
\end{abstract}

Key words: catch, commercial vessel, effort, fishery, Illex illecebrosus

\section{Introduction}

The I. illecebrosus fishery in the northwestern Atlantic Ocean is a trawl-based fishery. The gear used is typically one of several designs of otter trawl with a codend mesh size of $\geq 47.6 \mathrm{~mm}$ (Brodziak and Hendrickson, 1999; Dawe and Hendrickson, MS 1998; NEFSC, MS 1999). The fishery is managed using a 
yearly TAC (total allowable catch) (NEFSC, MS 1999). The vast majority of the squid are caught during the period of mid-June to early September (NEFSC, MS 1999; MAFMC, 1998; Hendrickson et al., MS 1996).

Illex illecebrosus normally lives less than one year (NEFSC, MS 1999). Unfortunately, the stock assessment process, commencing with one of several stock assessment surveys and ending with the establishing of a TAC, typically requires several years. As a consequence, the fishery is never prosecuted on the generation of squid providing the assessment data generating the TAC.

One obvious resolution to this dilemma is the utilization of real-time management. In this approach, information obtained from fishing vessels is applied in real time to determine at what point the fishery should be closed, thus regulating the catch. Real-time management is presently utilized in some squid fisheries (Agnew et al., 1998; Basson et al., 1996; Rosenberg et al., 1990; Morales-Bojórquez et al., 2001), but has not yet been implemented in any U.S. squid fishery. A key issue is the development of an approach to measure catch per unit effort (CPUE) during the course of the season without distributing an uneconomical number of observers within the fleet. With remote positioning technology, one can track a vessel remotely and input information provided by the Captain on catch and effort. This approach would be economical, if the accuracy of the Captains' reports can be confirmed by vessel tracking data and landings.

Any industry-based data collection program requires reassurance as to the accuracy of the reported data, however. Key to any real-time management program is the ability to confirm the accuracy of vesselreported data on effort, since landings can always be used to verify catch (but see Patterson, 1998). In 1999, 2000, and 2001, we collected vessel reports on tow position, tow duration, vessel speed, and water depth from fishing vessels fishing for I. Illecebrosus between Massachusetts and Virginia. These data were recorded by Captains as they fished and reported by FAX or e-mail. In 2001, a study was conducted onboard selected vessels to describe fishing behavior with specific emphasis on determining characteristic tow durations, tow distances, and tow speeds and the types of vessel behavior that occur between tows and during overnight periods when fishing does not take place (Powell et al., 2003). In the latter cases, the study targeted the discrimination of searching for another place to fish from other activities that might occur overnight or between tows, because searching may be an important component of effort. In this contribution, we apply these findings to the analysis of commerciallyreported data on tow position and tow duration to identify accuracy in vessel reporting and to further evaluate the influence of searching behavior in the distribution of vessel time allocation and in the calculation of CPUE.

\section{Methods}

\section{Data Collection}

Captains were asked to record information on each tow, including the position and time the net arrived on the bottom, the position and time the net left the bottom, the catch of I. illecebrosus for the tow, the beginning and ending depths for the tow, and the time spent searching. The majority of data returned for analysis had complete records, with one exception; Captains often failed to accurately record searching time. Accordingly, searching time has been estimated in an alternative way, as discussed later.

\section{Data Analysis}

Vessel time was allocated into one of five activities: searching, set-up time between tows, towing, steaming overnight, and laying-to overnight following Powell et al. (2003). Each was described by three metrics: distance traveled, duration, and vessel speed. Tow metrics were calculated directly from the empirical data returned by the Captains. Tow duration was defined as the difference between the time of net deployment and retrieval (in decimal hours). Tow 'straight-line' distance was defined as the linear distance from the reported positions of net deployment and retrieval. This metric was compared to the alternative estimate of tow distance obtained from tow duration and reported vessel speed. Reported vessel speed was also compared to tow speed over bottom ( $\mathrm{km}$ per $\mathrm{hr}$ ) calculated from tow duration and straight-line distance. Comparison was made to measurements obtained by scientist observers onboard selected vessels. This latter information has been more thoroughly reported in Powell et al. (2003).

Overnight periods were defined as the time from the last tow of the day until the first tow of the following day. Overnight periods were identified as overnight steams or periods laying-to based on distance traveled and vessel speed, as defined in Powell et al. (2003). Nighttime periods in which the vessel appeared to have steamed for part of the night were allocated proportionately to steaming and laying-to 
based on the comparison of vessel speed with a minimum defined steaming speed and a maximum defined laying-to speed from the observer study (Powell et al., 2003).

Similarly, periods between tows were defined as searches or time spent preparing for the next tow (setup time). These two activities could be unambiguously differentiated by their characteristic vessel speeds and distances traveled in observed tows (Powell et al., 2003). Searches were undertaken at significantly higher vessel speeds and covered significantly farther distances. Accordingly, the period between each specific tow was allocated to one of these two categories based on distance traveled and vessel speed. In some cases, the time between tows was characterized by an intermediate distance and speed. These ambiguous cases in which the vessel appeared to have searched for part of the time between tows were allocated proportionately to searching and tow preparation based on the comparison of vessel speed with a minimum defined searching speed and a maximum defined set-up speed from the observer study (Powell et al., 2003).

CPUE was calculated as catch $(\mathrm{kg})$ divided by effort. Effort was defined in several ways: duration of tow, straight-line distance of the tow determined from the positions of net deployment and retrieval as reported by the Captain, distance of tow determined from tow duration and vessel speed reported by the Captain, duration of tow including searching time, and distance of tow including searching distance. Searching distance was calculated from the position of net retrieval reported for the previous tow and the position of net deployment reported for the subsequent tow. This, consequently, is a minimum searching distance. Powell et al. (2003) observed that most searches were conducted as relatively straight-line transits. Cases where apparent speed between tows fell between the characteristic set-up and searching speeds might result from nonlinear searches. In this analysis, this alternative could not be differentiated from a combination of search and set-up. We have, therefore, relied on the observed tows reported by Powell et al. (2003) in choosing the latter explanation as the more likely.

We utilized principal components analysis (PCA) to create new variables that described components of vessel activity that tended to covary. For PCA analysis, the data were first standardized to a mean of zero and a standard deviation of 1 .
Statistical analysis used nonparametric ANOVA on ranked raw variables. Main effect variables in most tests included boat, year, and year-day. Year-day was defined as the daily increment from 1 January. In some cases, boats were grouped into categories based on vessel length or onboard storage methods. Storage methods included freezing (freezer trawlers) and refrigerated seawater (RSW trawlers). In most cases, the covariates depth and position were included. Depth was the average of the depths at net deployment and retrieval. Position was included as latitude (in decimal degrees). The I. illecebrosus fishery takes place at the edge of the continental shelf in the Mid-Atlantic from Massachusetts to Virginia (Fig. 1). Over much of this range, the continental shelf is oriented northeastsouthwest. Latitude, therefore, is an adequate comparator for position. Interaction terms between year and the other independent variables were routinely included in the model.

\section{Results}

\section{Descriptive Statistics}

Twelve boats fishing for I. illecebrosus reported data on catch and effort in 1999, six in 2000, and seven in 2001. In total, information on 2240 tows was obtained, 1146 tows in 1999,549 tows in 2000, and 545 tows in 2001. Of the 2240 tows, 1705 tows or $76.1 \%$ of the tows, were reported without apparent error in tow position and time of net deployment and retrieval. Reporting was most complete in 1999. Complete reports were obtained for $92.5 \%$ of the tows reported in 1999 , only $30.6 \%$ of the tows reported in 2000 , but reporting improved to $87.3 \%$ in 2001 . The vast majority of errors involved failure to report the ending position or ending time of the tow, so that effort could not be calculated in terms of both tow distance and tow duration. Much less frequently, inaccurate positions were reported that resulted in tow times or tow distances being impossibly long. A total of 16 tows out of 2240 (0.7\%) were disregarded for this reason.

The vast majority of tows occurred along the shelf edge from $40.6^{\circ} \mathrm{N} 67.1^{\circ} \mathrm{W}$ south and west to $34.7^{\circ} \mathrm{N}$ $74.0^{\circ} \mathrm{W}$ (Fig. 1). About 15 tows appear to have been conducted off the shelf edge. All of these tows occurred in 2000. Although the position of these tows is suspicious, careful perusal of the data did not reveal any reason to disbelieve the accuracy of the reports. These tows have been retained in further analyses.

Tows averaged $3.3 \mathrm{hr}$. Half of the tows fell within the range of 2.4 to $4.2 \mathrm{hr}$. Reported tow speeds 


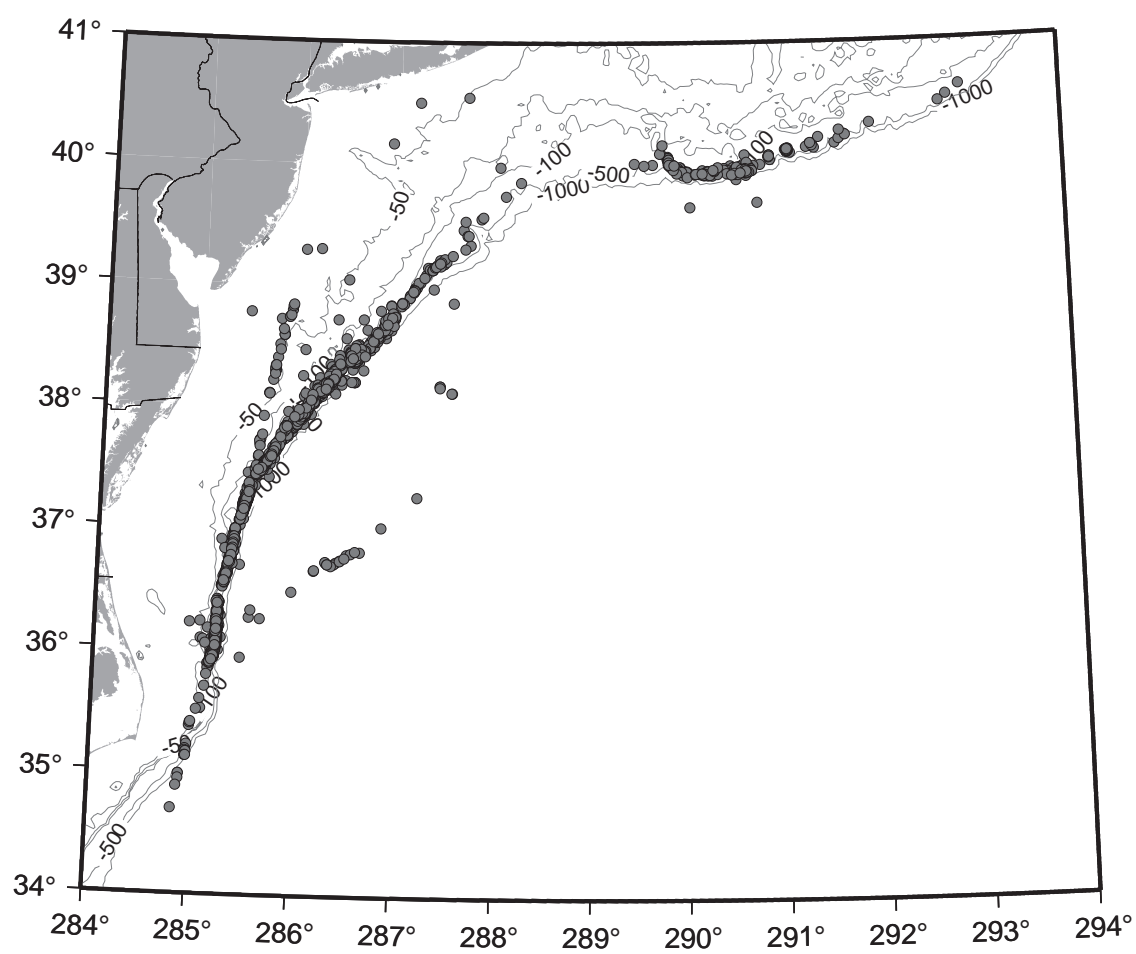

Fig. 1. Positions of tows reported by I. illecebrosus fishing vessels during 19992001 .

averaged $6.1 \mathrm{~km}$ per hr, with very little variation. Tow distances were calculated in two ways, as the straightline distance from the reported position of net deployment and retrieval and as the multiple of reported tow duration and reported vessel speed. The two estimates typically deviated by an average of $3 \mathrm{~km}$. So, tow paths frequently fell somewhat off a linear path. Straight-line distances for tows averaged $17.4 \mathrm{~km}$, so the average deviation between the two methods for estimating distance amounted to about $17 \%$.

The difference in the two estimates of tow distance arises because some tows did not follow a linear path (Fig. 2). For nine of 14 boats, this deviation was significantly different from zero (two-tailed t-test, $a=$ 0.05 ). For 8 of these 9 boats, the deviation was positive, indicating that the boat traversed a nonlinear path while towing. For the ninth boat, the deviation was negative, suggesting that this boat routinely underreported tow duration or tow speed.

Two alternative interpretations arise, however, for the deviations in tow distance. In the one case, biased high-reporting of vessel speed would produce a positive deviation indistinguishable from that produced by a nonlinear path. In the second case, consistent towing with a current would mimic a nonlinear path. The few negative deviations, only one boat consistently reported data with negative deviations (Fig. 2), indicate that the converse of both alternative explanations must have occurred rarely, a consistency that might not be expected across the entire reporting fleet, over all depths, and throughout the geographic range. The large bias favoring positive deviations, then, suggests that nonlinear towing is the most reasonable explanation and this agrees with similar results from observed tows (Powell et al., 2003).

Tow distance, tow duration, and tow speed varied significantly among boats and between years (Table 1, Fig. 3-4). Tow durations were longest in 2000 and shortest in 2001. However, mean tow durations only ranged from 3.19 to $3.42 \mathrm{hr}$. Tow distances were longer in 2000 than in the other two years. Tow distances averaged $23.3 \mathrm{~km}$ in 2000 and only 15.9 and $17.7 \mathrm{~km}$ in 2001 and 1999, respectively. Tow deviations, the difference between tow distance measured as a straight line between the positions of net deployment and retrieval and the distance estimated from tow duration and reported vessel speed, were highest in 2001 and lowest in 1999. Interaction terms between year and the other independent variables were routinely significant. In particular, individual boats operated at somewhat different speeds and towed for somewhat different 


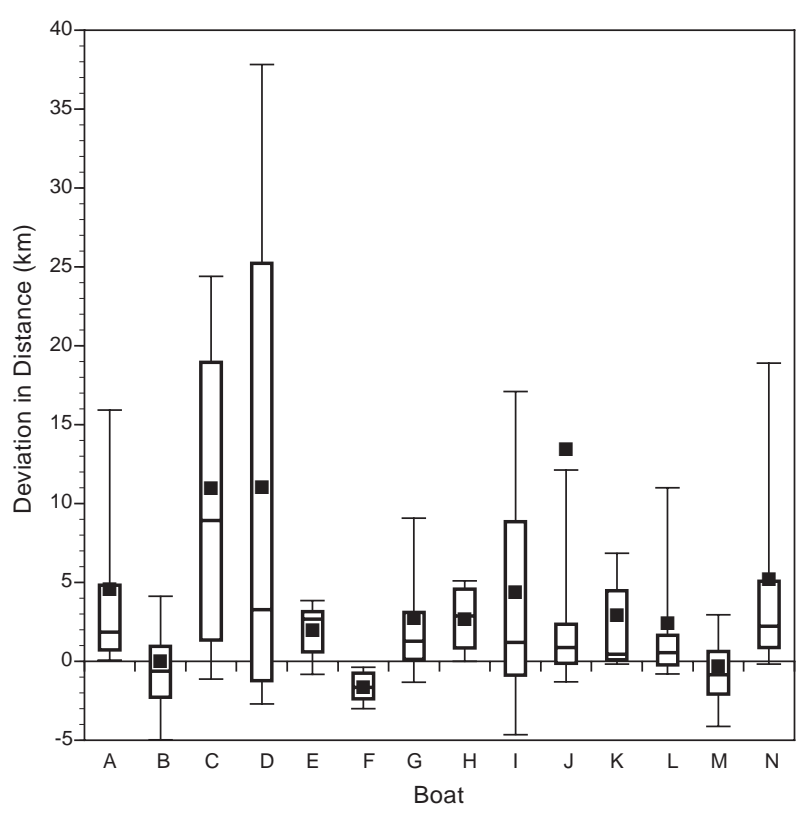

Fig. 2. Deviation in tow path from a straight line as deduced from the difference in the distance between net deployment and net retrieval and the distance computed from tow duration and reported vessel speed $(\Delta \mathrm{km})$. Positive deviations indicate a nonlinear path. Negative deviations indicate some error in the accuracy of reported positions, times, or vessel speeds. Box boundaries are the interquartile range split by the median. Whiskers encompass $90 \%$ of the data. The mean is shown by a

distances between years (Table 1). Also of note, tow duration varied between years with depth and latitude. Very likely, these differences come about because I. illecebrosus were distributed differentially between years with respect to depth and latitude.

\section{Searching Behavior}

Originally, the Captains were requested to provide information on searching time before each tow. Unfortunately, Captains failed to consistently distinguish searching from other activities that occurred between tows. However, observed tows identified criteria that could be used to distinguish searching behavior from other between-tow activities (Powell et al., 2003). Of particular importance was vessel speed. Searching normally took place at a vessel speed of $\geq 13 \mathrm{~km}$ per hr whereas other activities took place at much lower vessel speeds. In addition, searches took relatively linear paths. Consequently, we used information on distance traveled between the location of net retrieval and the next deployment to reconstruct vessel speed and used that information to identify between-tow periods where searching occurred.

Individual boats searched prior to $10 \%$ to $50 \%$ of the tows depending upon the boat. Searching occurred prior to $30.0 \%$ of the tows reported in $1999,20.4 \%$ of the tows in 2000 , and $39.0 \%$ of the tows in 2001 . Vessels averaged $1.7 \mathrm{hr}$ searching, when searching occurred, covering an average distance of $23.3 \mathrm{~km}$ (Fig. 5). Searching time was about one-half of the average duration of a tow and searching distance was about $115 \%$ of tow distance. Searching behavior was not significantly different between boats or years, nor did it vary much during the year (Table 1).

If successful, searching should improve subsequent catch. Searching duration did not significantly influence subsequent catch. Searching distance moderately influenced subsequent catch (ANOVA, $P=0.02$ ). However, highest searching times were associated with low subsequent catches (Fig. 6). We compared the catches before and after a search with the catches before and after set-up periods during which time no searching occurred. If searching is successful, the differential in catch before and after searching events should exceed that before and after non-searching events. However, catch did not improve significantly after a search in comparison to other between tow activities, nor did an increase in the duration of a search increase the differential in catch (ANOVA, $a=0.05$, Fig. 7).

\section{Catch-per-Unit Effort}

Catch averaged $5130 \mathrm{~kg}$ per tow. Catch varied significantly by year (Table 2). Catch was lowest in $2001,4751 \mathrm{~kg}$ per tow and highest in 2000,5 $471 \mathrm{~kg}$ per tow. Catch varied significantly between boats (Fig. 8), but was little influenced by tow depth or the latitude that fishing occurred. Interaction terms with year were highly significant (Table 2), indicating that the catch of individual boats varied differentially over the three year period and that the locations and depths fished varied between the years. Time of year (year-day) contributed only moderately to variation in catch (Table 2).

CPUE was calculated with and without searching included in the effort component. CPUE was based either on distance traveled during a tow or tow duration. As noted earlier, the straight-line estimate of tow distance obtained from the Captains' reports of the positions of net deployment and retrieval generally underestimated true tow distance. All four measures of 
effort, tow distance, tow duration, tow + search distance, and tow + search duration, yielded approximately the same trends. CPUE by any measure varied significantly between boats and among years (Fig. 9, Table 2). CPUE was rarely significantly influenced by depth or latitude and never by time of year (Table 2). Interaction terms with year were routinely significant, indicating that the influence of boat, time of year, and depth on CPUE varied between years. Most noteworthy is the stronger influence of the interaction between year and time of year and depth and year in comparison to the variables as main effects.

TABLE 1. Significance levels from ANCOVA analysis. Dependent variables include the straight-line distance computed from the reported positions of net deployment and retrieval, the tow duration computed from the reported times of net deployment and retrieval, the reported tow speeds, the difference between the tow distance computed from reported speed and tow duration and the straight-line estimate, the estimated straight-line searching distance, and the estimated searching duration. Significance is indicated as $P<$ the given P-value. - , not significant at $\alpha=0.05$. *indicates an interaction term.

\begin{tabular}{|c|c|c|c|c|c|c|}
\hline & $\begin{array}{l}\text { Straight } \\
\text { Line } \\
\text { Distance }\end{array}$ & $\begin{array}{c}\text { Tow } \\
\text { Duration }\end{array}$ & $\begin{array}{c}\text { Tow } \\
\text { Speed }\end{array}$ & $\begin{array}{c}\text { Estimated } \\
\text { Deviation in } \\
\text { Tow Distance }\end{array}$ & $\begin{array}{l}\text { Searching } \\
\text { Distance }\end{array}$ & $\begin{array}{l}\text { Searching } \\
\text { Duration }\end{array}$ \\
\hline Boat & 0.0001 & 0.0001 & 0.0001 & 0.0001 & - & - \\
\hline Year & 0.001 & 0.0001 & - & 0.007 & - & - \\
\hline Year-day & - & - & 0.01 & 0.03 & - & - \\
\hline Depth & - & - & 0.02 & - & - & - \\
\hline Latitude & - & - & 0.03 & 0.008 & - & - \\
\hline Boat*Year & 0.0001 & - & 0.0001 & 0.007 & - & - \\
\hline Year-day*Year & - & 0.02 & 0.02 & 0.02 & - & - \\
\hline Depth*Year & - & 0.0001 & 0.0005 & - & - & - \\
\hline Latitude*Year & 0.0001 & 0.0001 & 0.05 & - & - & - \\
\hline
\end{tabular}

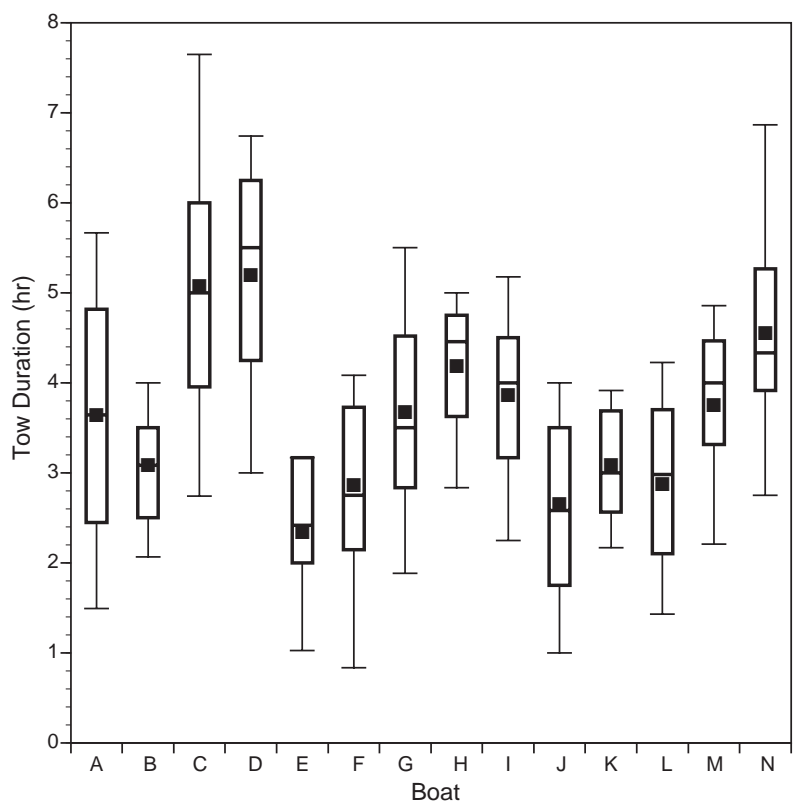

Fig. 3. Tow duration (hr) as it varied among boats. Box boundaries are the interquartile range split by the median. Whiskers encompass $90 \%$ of the data. The mean is shown by a

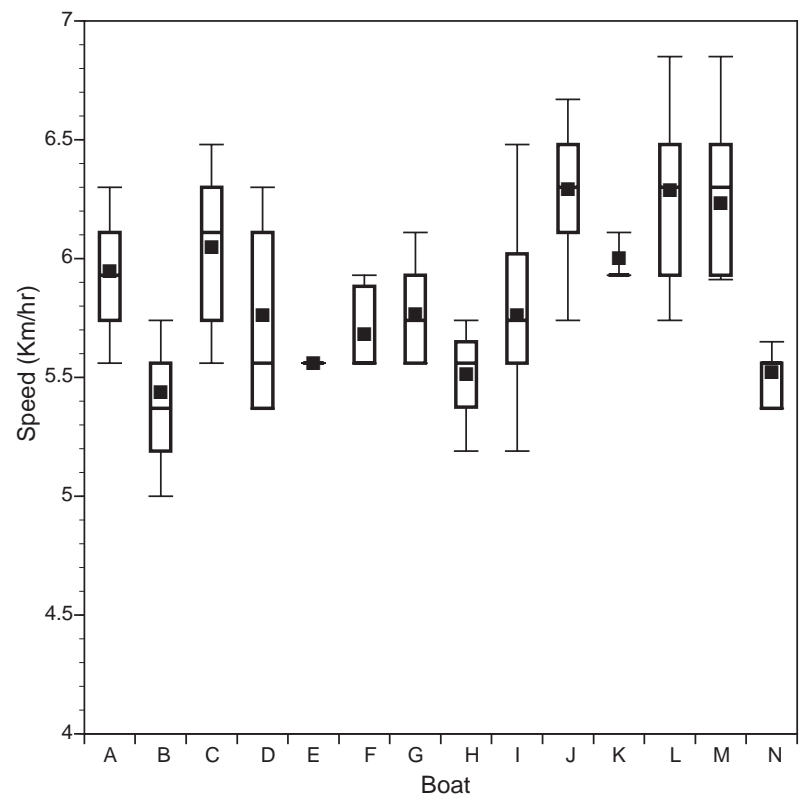

Fig. 4. Reported tow speed (km per hr) as it varied among boats. Box boundaries are the interquartile range split by the median. Whiskers encompass $90 \%$ of the data. The mean is shown by a 


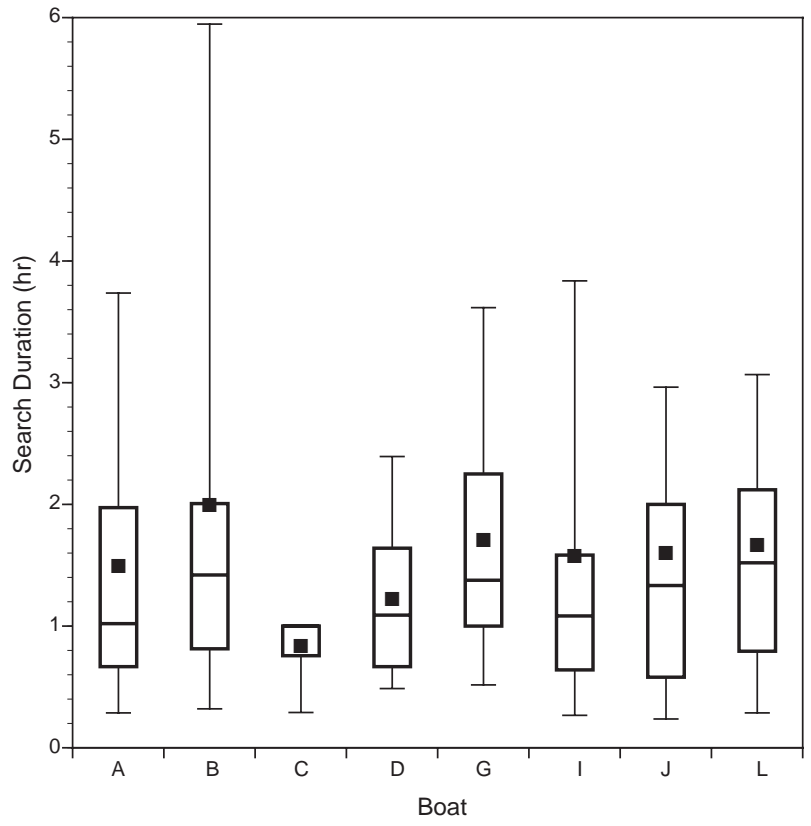

Fig. 5. Search duration (hr) as it varied among boats for boats with $>3$ searches. Box boundaries are the interquartile range split by the median. Whiskers encompass $90 \%$ of the data. The mean is shown by a
Also noteworthy is the essential absence of an effect of latitude on CPUE either as a main effect or in an interaction term.

Catch was significantly correlated with CPUE (Fig. 10), regardless of the effort term used to calculate CPUE and regardless of whether searching was included in the effort term. Using tow duration, rather than tow distance, as a measure of effort produced higher regression coefficients. When examined by ANOVA, catch was not significantly influenced by tow duration, tow distance, year-day, or the interaction between yearday and either of the two effort measures. Finally, the frequency at which a boat searched might bear some relationship to CPUE if searching successfully increased CPUE. However, searching frequency (number of searches per number of tows) was not significantly related to CPUE (Spearman's rank correlation, $a=0.05$ ).

\section{Overnight Transits}

Boats also transited overnight on some nights. Such transits might be a type of searching behavior conducted when the activity does not restrict fishing

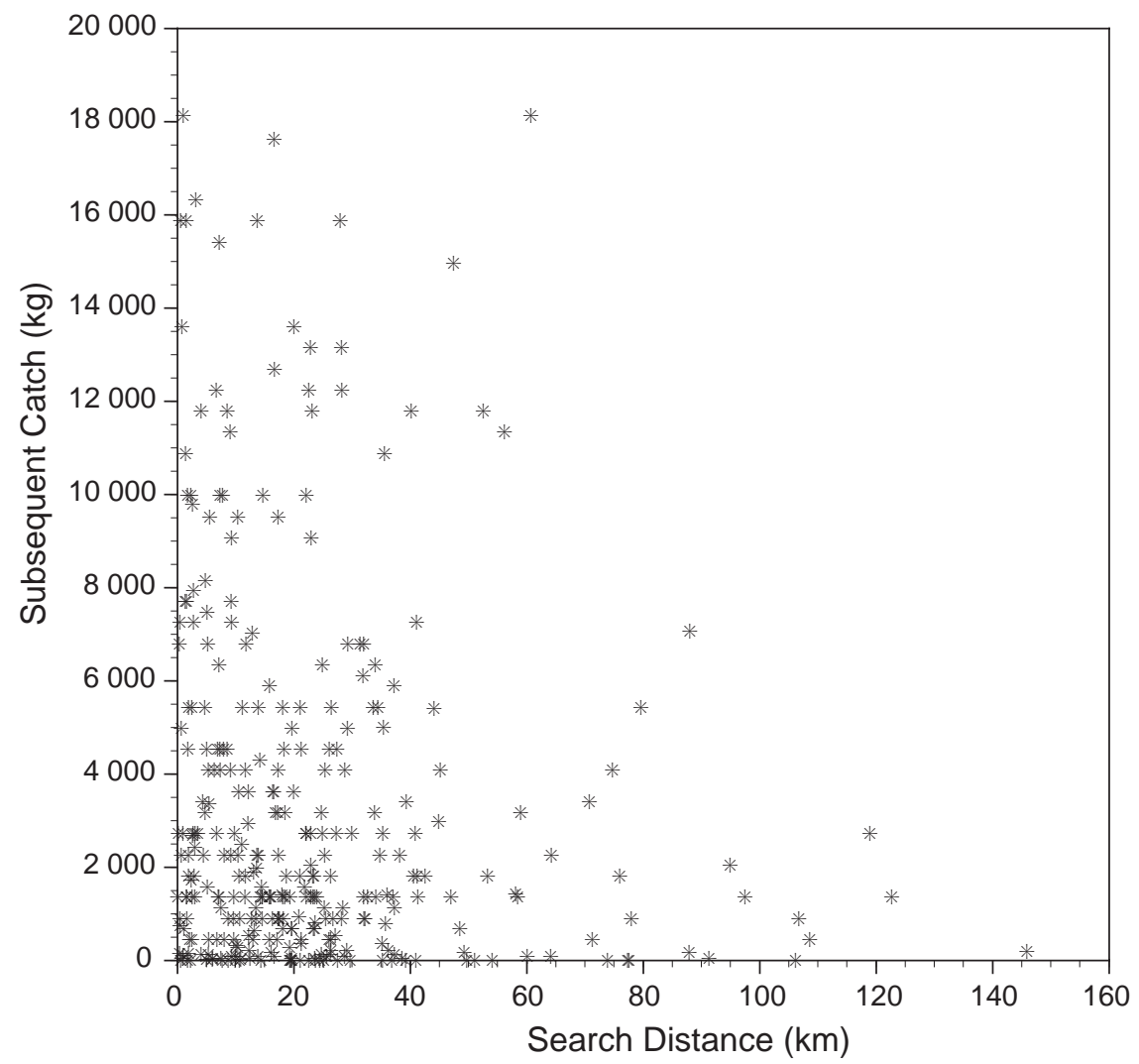

Fig. 6. Search distance $(\mathrm{km})$ versus catch on the subsequent tow $(\mathrm{kg})$ for the 14 vessels participating in the data collection program during 1999-2001. 


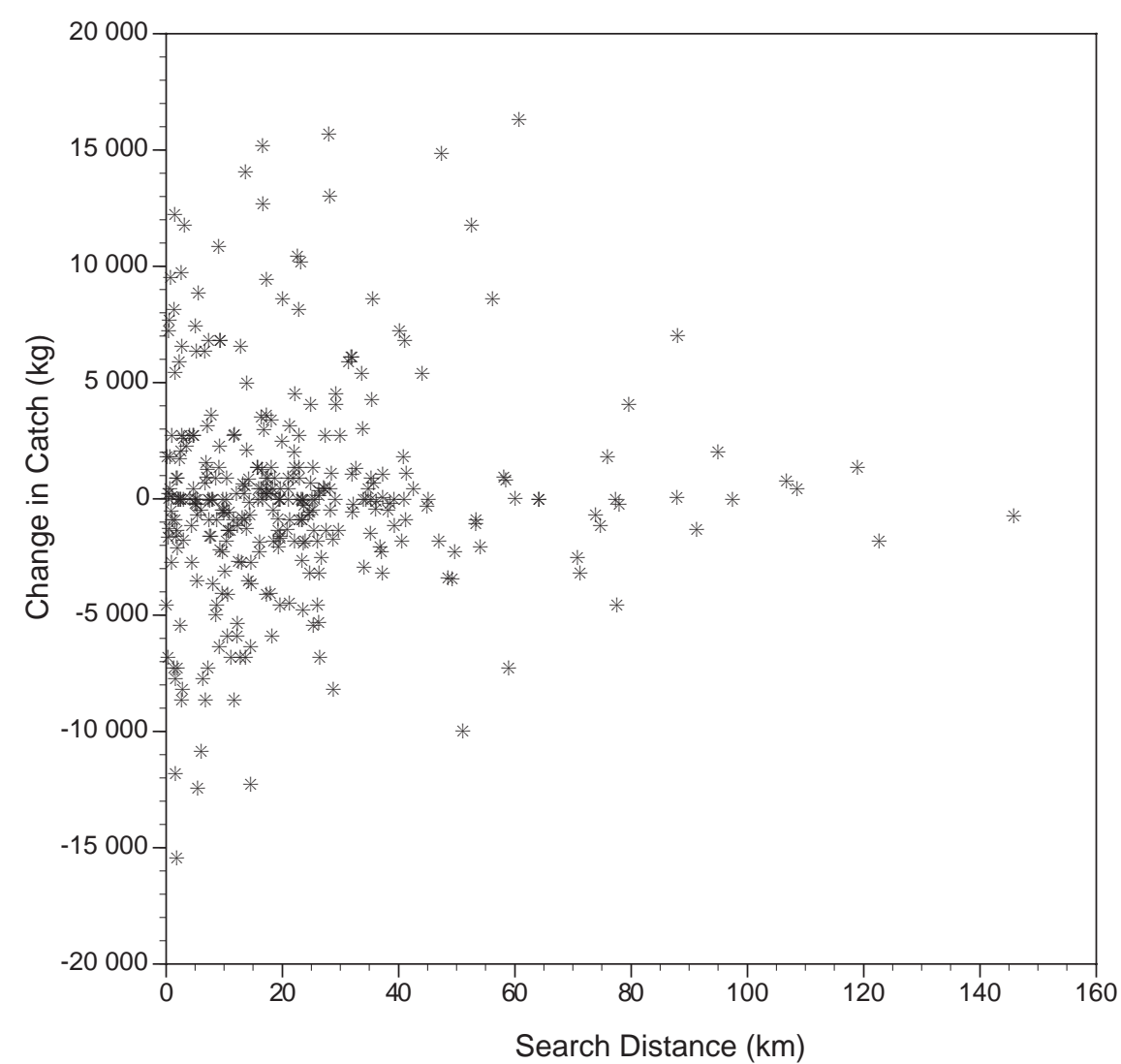

Fig. 7. Search distance $(\mathrm{km})$ versus the change in catch between the prior and subsequent tow $(\mathrm{kg})$ for the 14 vessels participating in the data collection program during 1999-2001. A positive deviation indicates that catch increased after the search.

TABLE 2. Significance levels from ANCOVA analysis. Dependent variables include catch, CPUE as a function of tow distance, CPUE as a function of tow duration, CPUE as a function of the sum of tow and search distance, and CPUE as a function of the sum of tow and search duration. All distances are straight-line distances. Significance is indicated as $P<$ the given $P$-value. - , not significant at a $=0.05$. ${ }^{*}$ indicates an interaction term.

\begin{tabular}{lccccc}
\hline \hline & & $\begin{array}{c}\text { Tow } \\
\text { Distance Determined } \\
\text { CPUE }\end{array}$ & $\begin{array}{c}\text { Tow } \\
\text { Duration Determined } \\
\text { CPUE }\end{array}$ & $\begin{array}{c}\text { Tow and Search } \\
\text { Distance Determined } \\
\text { CPUE }\end{array}$ & $\begin{array}{c}\text { Tow and Search } \\
\text { Duration Determined } \\
\text { CPUE }\end{array}$ \\
\hline Boat & 0.0001 & 0.0001 & 0.0001 & 0.0001 & 0.0001 \\
Year & 0.0001 & 0.0001 & 0.0001 & 0.0001 & 0.0008 \\
Year-day & 0.01 & - & - & - & - \\
Depth & - & - & 0.0002 & - & - \\
Latitude & 0.03 & - & 0.05 & 0.002 & 0.01 \\
Boat*Year & 0.0005 & 0.001 & 0.005 & 0.003 & - \\
Year-day*Year & 0.0001 & 0.0001 & 0.0001 & - & - \\
Depth*Year & 0.0001 & 0.0008 & 0.0001 & - & - \\
Latitude*Year & 0.002 & - & - & & - \\
\hline
\end{tabular}




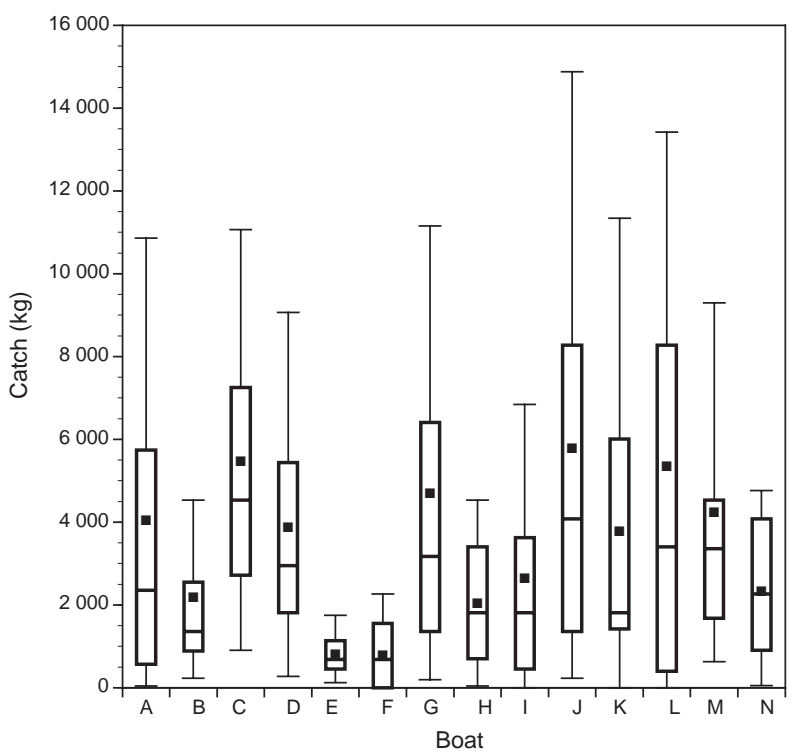

Fig. 8. Catch per tow (kg) as it varied among boats. Box boundaries are the interquartile range split by the median. Whiskers encompass $90 \%$ of the data. The mean is shown by a

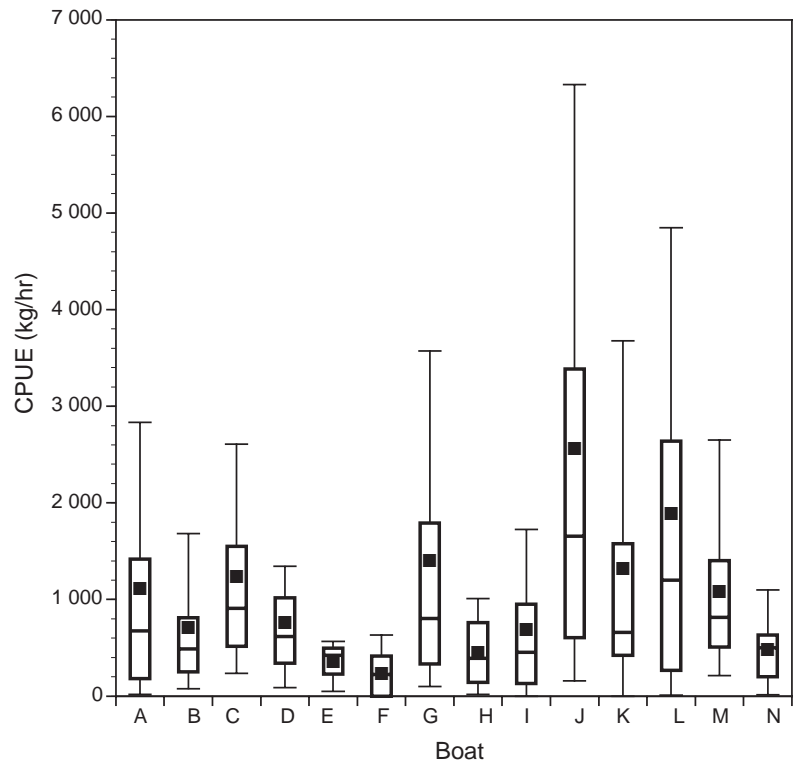

Fig. 9. Catch per unit effort based on the duration of the tow (kg per hr) as it varied among boats. Box boundaries are the interquartile range split by the median. Whiskers encompass $90 \%$ of the data. The mean is shown by a

time. The data set contained 420 overnight periods. Transiting occurred in 124 such periods, about $30 \%$ of the time. This ratio was very similar to the ratio of searches to total tows.
The distance traveled in overnight transits was not significantly related to the subsequent day's catch (Spearman's rank correlation, $a=0.05$ ). Whether or not the boat steamed overnight did not significantly influence the day's subsequent catch. Interaction terms with boat, year, and year-day were also not significant (ANOVA, $a=0.05$ ). Accordingly, overnight transits did not appear to influence catch.

\section{Boat Types}

Illex illecebrosus trawlers are either freezer trawlers or RSW (refrigerated seawater) trawlers. Fishing trips are shorter in duration for RSW trawlers because onboard storage time is limited. Differences in tow duration, reported tow speed, and estimated tow distance (from reported speed and duration), were highly significantly different for these two boat types (ANOVA, Table 3). Tows on RSW trawlers deviated more from a straight-line path. Catch per tow was higher for the freezer trawlers, as was CPUE (Table 3). Interactions with year were significantly different for most tow metrics, including tow distance, tow duration, and tow speed, indicating that the differences observed between these two vessel classes varied between years. CPUE, with effort determined by tow duration, was significantly higher for freezer trawlers than for RSW trawlers (Table 3). This variation was produced, first, by tow durations that averaged $25 \%$ shorter in duration on freezer trawlers and, second, by tow catches that averaged about $25 \%$ larger for freezer trawlers. However, interactions with year were not significant, indicating that the difference between the two boat types in catch and CPUE was relatively stable over the three years. Finally, freezer trawlers searched about twice as often as RSW trawlers. No evidence exists suggesting that searching influenced the larger CPUE on freezer trawlers, although searching occurred more frequently on these vessels.

Vessels varied considerably in length. About half of the vessels reporting data for this analysis were over $30 \mathrm{~m}$ in length. Accordingly, we compared two groups of vessels, large and small, defined by a $30-\mathrm{m}$ length discriminator. The two size-classes of vessels operated significantly differently from the standpoint of tow duration, tow distance, and tow speed (Table 4). The larger vessels' tows deviated more from a straight line. Catch-per-tow was significantly higher for these larger vessels, as was CPUE (Table 4). Interactions with year were significant only for a few tow characteristics: tow duration, tow speed, and catch per tow. CPUE did not have a significant interaction term with year, nor did any measure of tow distance 


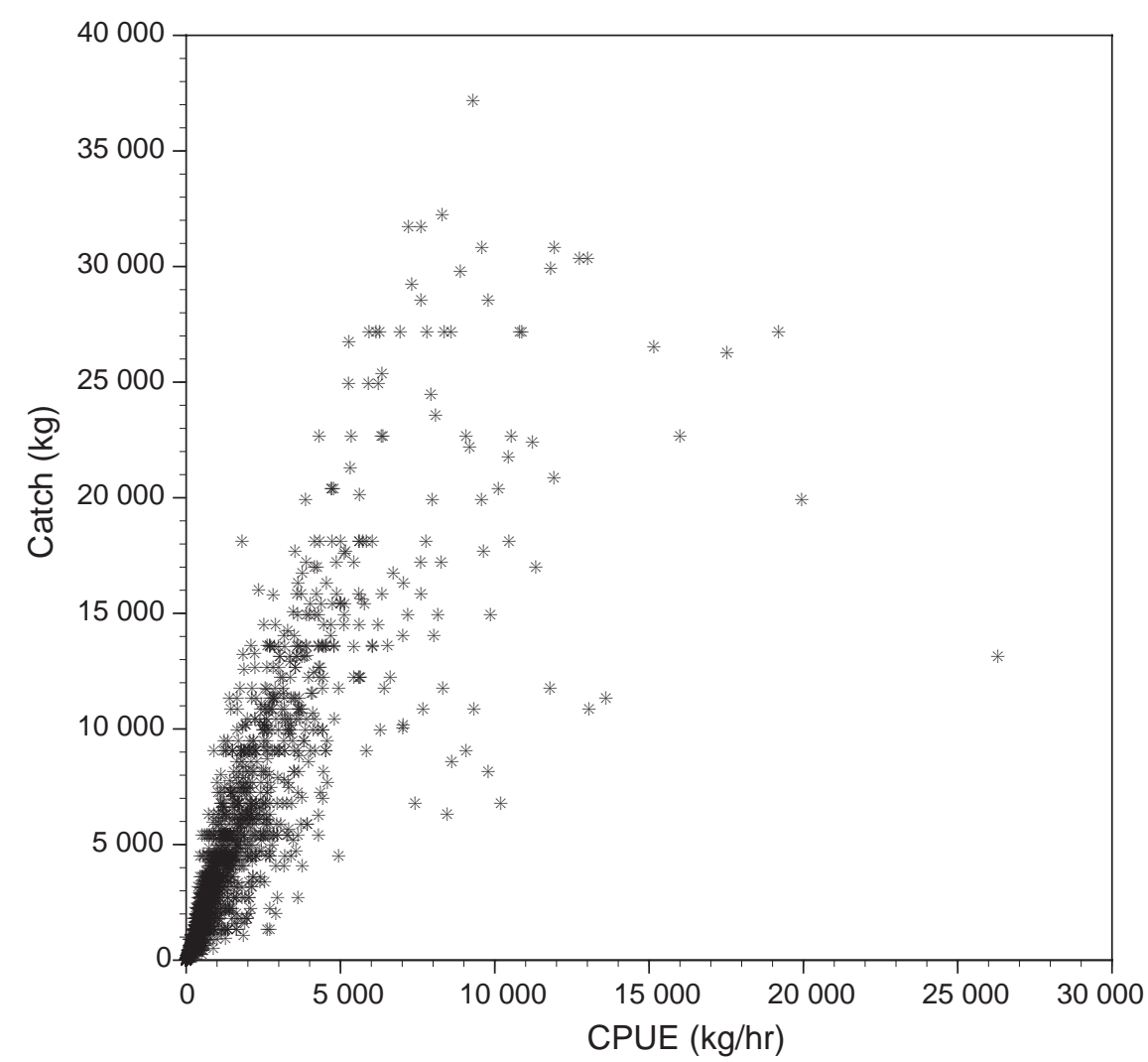

Fig. 10. The relationship between catch $(\mathrm{kg})$ and catch per unit effort ( $\mathrm{kg}$ per hr) with effort defined in terms of the duration of a tow.

TABLE 3. Comparison of tow characteristics for freezer trawlers and refrigerated seawater (RSW) trawlers. Straight-line distance was estimated from the beginning and ending positions for tows. Deviation in distance is the difference of that estimate from one made using the duration of the tow and the Captains' reported vessel speed. Significance levels from ANOVA analysis comparing RSW trawlers with freezer trawlers with year as the other main effect. Significance is indicated as $P<$ the given $P$-value. - , not significant at a $=0.05$.* indicates an interaction term.

\begin{tabular}{lccrr}
\hline \hline & \multicolumn{2}{c}{ Refrigerated } \\
& Freezer trawler & Seawater Trawler & RSW & RSW*Year \\
\hline Straight-line tow distance $(\mathrm{km})$ & 16.8 & 19.9 & 0.0001 & 0.01 \\
Tow duration $(\mathrm{hr})$ & 3.1 & 4.3 & 0.0001 & 0.0001 \\
Tow distance $(\mathrm{km})$ & 19.2 & 24.8 & 0.0001 & 0.0002 \\
Tow speed $(\mathrm{km}$ per hr) & 6.1 & 5.7 & 0.0001 & 0.0001 \\
Deviation in distance $(\Delta \mathrm{km})$ & 2.5 & 5.2 & 0.0001 & - \\
Catch (kg per tow) & 5335 & 3814 & - & - \\
Catch per hour $(\mathrm{kg}$ per hr) & 1791 & 921 & 0.0006 & - \\
Catch per distance $(\mathrm{kg}$ per km) & 690 & 540 & - & - \\
Search frequency $(\%)$ & 36.6 & 17.7 & - & - \\
\hline
\end{tabular}

(Table 4). Finally, larger vessels searched about twice as often as smaller vessels. No evidence exists suggesting that searching influenced the larger CPUE on larger vessels, although searching occurred more frequently on these vessels.
PCA analysis was used to generate composite scores describing combinations of vessel behavior to better evaluate why these vessel classes differed significantly in ANOVA analysis. PCA analysis revealed that both sets of boat categories, RSW versus 
TABLE 4. Comparison of tow characteristics for vessels above and below $30 \mathrm{~m}$ in length. Straight-line distance was estimated from the beginning and ending positions for tows. Deviation in distance is the difference of that estimate from one made using the duration of the tow and the Captains' reported vessel speed. Significance levels from ANOVA analysis comparing large and small trawlers with year as the other main effect. Significance is indicated as $P<$ the given $P$-value. - , not significant at $a=0.05$. *indicates an interaction term.

\begin{tabular}{lrrrr}
\hline \hline & $\begin{array}{c}\text { Vessels } \\
<30 \mathrm{~m}\end{array}$ & $\begin{array}{r}\text { Vessels } \\
>30 \mathrm{~m}\end{array}$ & Length & Length*Year \\
\hline Straight-line tow distance $(\mathrm{km})$ & 18.7 & 17.1 & 0.02 & - \\
Tow duration (hr) & 3.28 & 3.30 & 0.002 & 0.0001 \\
Tow distance $(\mathrm{km})$ & 18.4 & 20.3 & 0.0001 & - \\
Tow speed $(\mathrm{km} \mathrm{per} \mathrm{hr)}$ & 5.6 & 6.1 & 0.0001 & 0.0001 \\
Deviation in distance $(\Delta \mathrm{km})$ & -0.2 & 3.4 & 0.0001 & - \\
Catch (kg per tow) & 2.4 & 5.5 & 0.0001 & 0.002 \\
Catch per hour (kg per hr) & 713 & 1842 & 0.0001 & - \\
Catch per distance $(\mathrm{kg}$ per km) & 195 & 764 & 0.0001 & - \\
Search frequency $(\%)$ & 13.4 & 35.5 & & \\
\hline
\end{tabular}

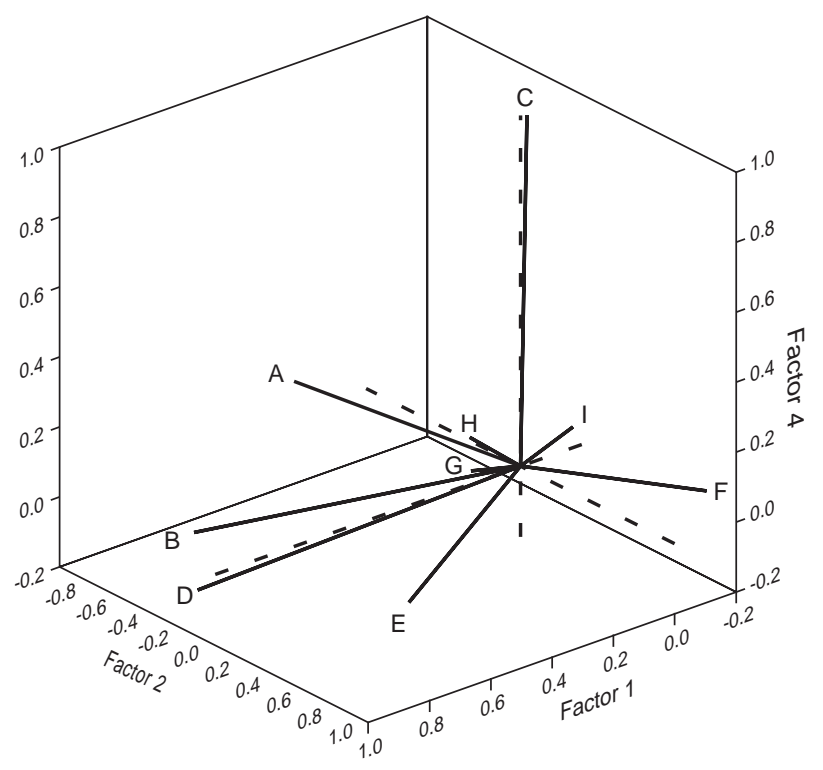

Fig. 11. Factor loading chart for PCA factor variables 1,2 and 4. (A) deviation between straight-line tow distance and true tow distance; (B) tow distance estimated from Captains' reported speed and tow duration; (C) Captains' reported speed; (D) tow duration; (E) straight-line tow distance; (F) tow speed estimated from tow duration and straightline distance; (G) catch; (H) CPUE with effort defined by straight-line tow distance; (I) CPUE with effort defined by tow duration.

freezer and large versus small, could best be separated using measures of (a) tow duration and true tow distance estimated from Captains' reports of tow duration and vessel speed (Factor 1, Fig. 11), (b) straight-line distance estimated from Captain's reported positions of tow initiation and cessation, tow speed estimated from straight-line distance and tow duration, and the deviation of tow distance as estimated from straight-line distance and from the Captain's reported speed and tow duration (Factor 2, Fig. 11), and (c) reported vessel speed (Factor 4, Fig. 11). The vessel groups defined by length and onboard storage strategy could be readily separated in PCA factor space using the same three factor variables. Tow duration and true distance traveled were most responsible for the separation of freezer trawlers and RSW trawlers (Fig. 12). PCA analysis also reveals the much greater variability in tow characteristics for the RSW trawlers than for the freezer trawlers. Reported tow speed and the deviation from a straight-line path were most responsible for the separation of large and small vessels (Fig. 13). Smaller vessels were not obviously characterized by greater or lesser variability in these characteristics, however.

\section{Discussion}

The purpose of this study was to investigate the validity and utility of data reported by commercial vessels for application in real-time management in the I. illecebrosus squid fishery in the Mid-Atlantic Bight. Assessing utility includes evaluating the frequency of erroneous reports, identifying the best measures of effort for CPUE analysis, and examining the influence of vessel type and vessel behavior on subsequent analyses of trends in catch and effort. 


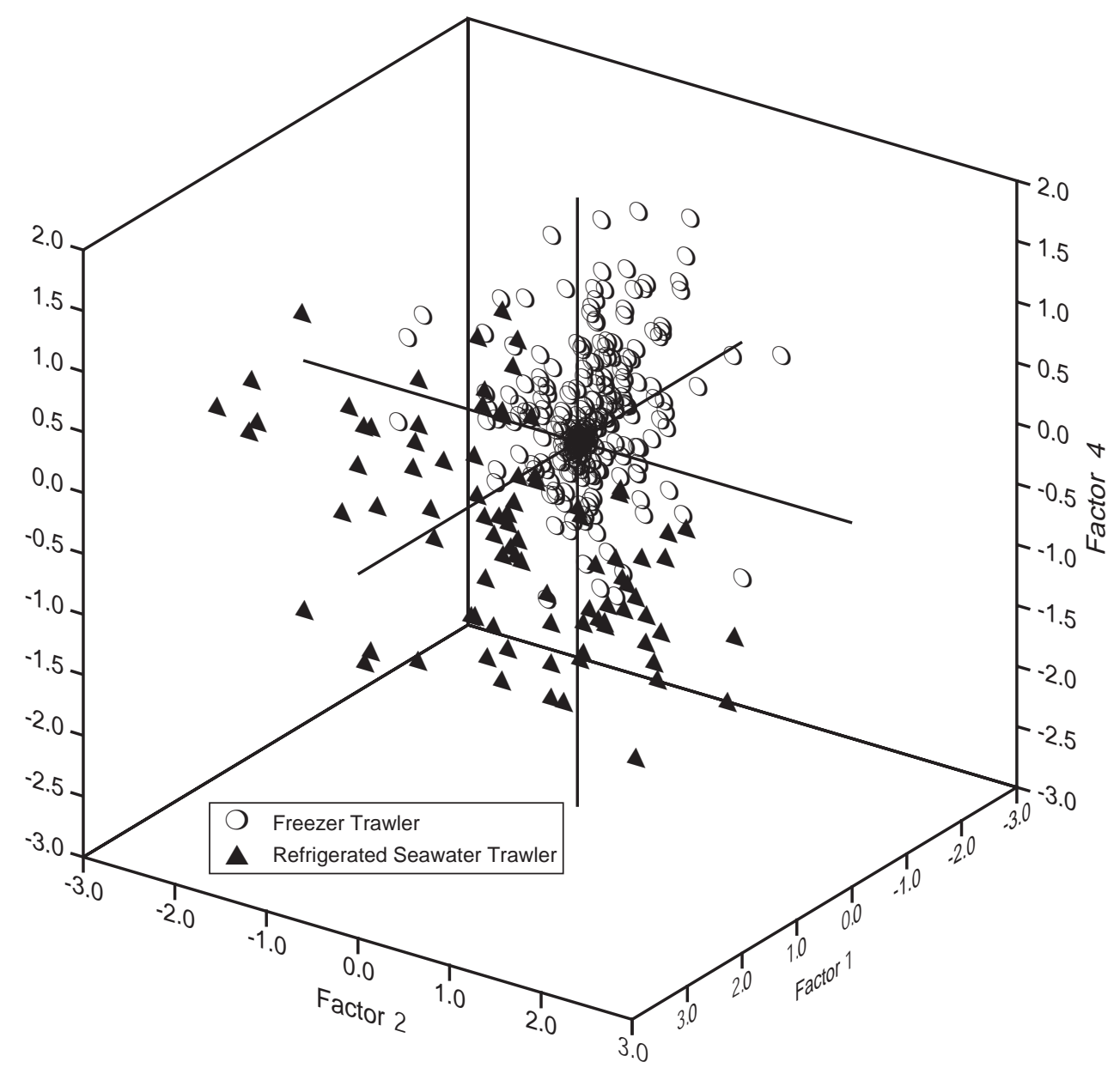

Fig. 12. PCA factor daily-mean scores for each of the three years for the trawlers using the two onboard storage methods, RSW and freezer.

\section{Reporting Errors}

Errors of three kinds existed in the commercial data. First, in about $24 \%$ of the reported tows, one or more key data items were missing. The vast majority of these omissions was either the ending time of the tow or the ending position of the tow. Second, tow positions or times were incorrectly reported. These errors occurred much less frequently, less than $1 \%$ of the time. Misreported times and positions were identified by unusual tow durations, distances, or speeds based on a subset of observed tows from 2001. These observed tows were analyzed fully by Powell et al. (2003).

Third, Captains were asked to include time spent searching between tows in their reports. The vast majority of these reported data could not be used because either the Captain failed to report the datum at all or because the Captain failed to accurately distinguish between searching and other activities that occur between tows. Observed trips demonstrated that searches could be distinguished clearly from other between-haul activities by vessel speed and distance traveled (Powell et al., 2003). Initial evaluation of the larger data set of reported tows considered here using the distances and times between one net retrieval and the next net deployment showed that this dichotomy existed as well in the Captain-reported data set. Therefore, the analytical pathway chosen for this study was to reconstruct between-tow activities from the more accurately-reported data on tow times and tow positions. That approach worked well. Average search distances and durations fell very close to the observed subset of tows (Table 5). Further, other activities between tows also fell within a similar range. In observed tows, these other activities typically took $0.5 \mathrm{hr}$ and the vessel traveled about $2.3 \mathrm{~km}$. In the larger 


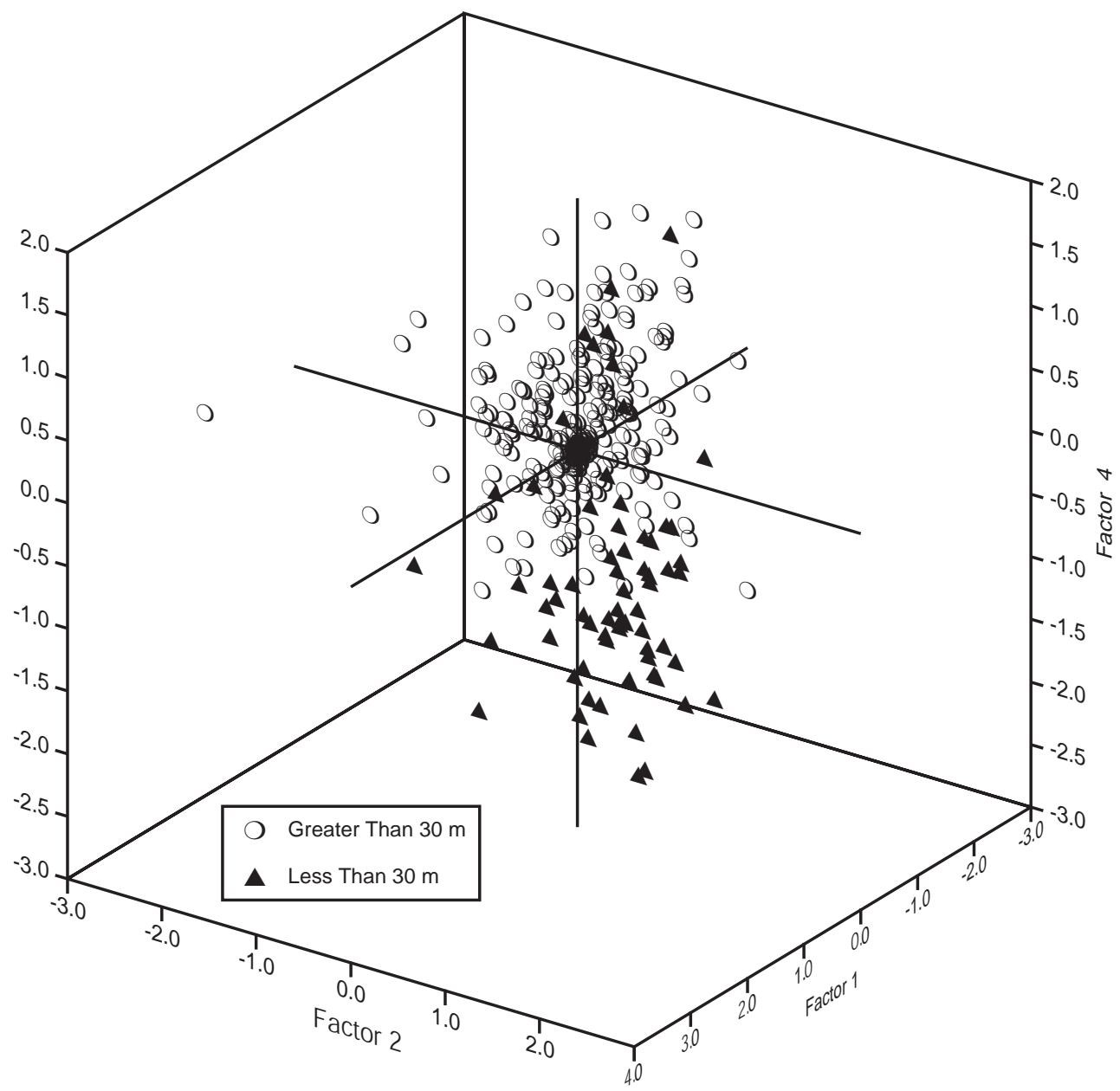

Fig. 13. PCA factor daily-mean scores for each of the three years for the two vessel size classes, $<30 \mathrm{~m}$ and $>30 \mathrm{~m}$.

data set, the values were somewhat higher at $1.4 \mathrm{hr}$ and $3.3 \mathrm{~km}$; however, the observed tows distinguished between time spent preparing for that next tow and processing time after a particularly large tow. Those two activities could not be distinguished a posteriori from tow times and positions and probably led to the somewhat increased amount of time spent in these activities as estimated from the larger Captain-reported data set.

Utilization of the observed tows as a basis for reconstruction of searching behavior is premised on the expectation that observed tows are representative of all tows. Such a comparison is provided in Table 5 . Tow speed, tow duration, and tow distance were in very close agreement between the two data sets. Tow straight-line distance was somewhat shorter in the observed trips, although still similar to the larger data set. Search distances and times were also very similar.
The only activity that diverged considerably was overnight transits. Overnight transits on the 2001 observed trips were twice as long in duration and more than four times as long in distance as in the Captainreported data set. Despite this latter difference, the Captain-reported data diverged sufficiently little from the observed trips to validate the general accuracy of the reports and also to suggest that vessel activities on observed trips were essentially identical to vessel activities on unobserved trips.

\section{Straight-Line versus Real Distances}

Straight-line distances can be calculated from reported positions of net deployment and retrieval. Vessels typically tow in a relatively straight line. However, true distances were routinely underestimated by $10-20 \%$ by this method, using time of net deployment and retrieval to measure tow duration and the Captain-reported vessel speeds. Observer data 
TABLE 5. Comparison of all activities for year's 1999, 2000, and 2001, as estimated from Captains' reports, with a subset of observed tows taken in 2001 and reported upon by Powell et al. (2003).

\begin{tabular}{|c|c|c|}
\hline & This Study & 2001 Observed Tows \\
\hline Straight-line tow distance $(\mathrm{km})^{1}$ & 17.4 & 15.6 \\
\hline Tow duration $(\mathrm{hr})^{1}$ & 3.3 & 3.2 \\
\hline Tow distance $(\mathrm{km})^{1}$ & 20.4 & 19.4 \\
\hline Tow speed $(\mathrm{km} \text { per hr})^{2}$ & 6.1 & 6.1 \\
\hline Set-up distance $(\mathrm{km})^{3}$ & 3.3 & 2.3 \\
\hline Set-up duration $(\mathrm{hr})^{3}$ & 1.4 & 0.5 \\
\hline Search distance $(\mathrm{km})^{3}$ & 23.3 & 25.6 \\
\hline Search duration $(\mathrm{hr})^{3}$ & 1.7 & 1.6 \\
\hline Straight-line overnight transit distance $(\mathrm{km})^{3}$ & 34.9 & 134.1 \\
\hline Overnight transit duration ${ }^{3}$ & 5.5 & 10.8 \\
\hline Straight-line overnight lay-to distance $(\mathrm{km})^{3}$ & 13.5 & 12.2 \\
\hline Overnight lay-to duration $(\mathrm{hr})^{3}$ & 10.0 & 9.1 \\
\hline
\end{tabular}

1 Calculated from Captains' reports for this study.

2 Estimated from reported straight-line tow distances and tow durations for this study.

3 Estimated from estimated speeds, using criteria developed by Powell et al. (2003), for this study.

indicates that actual vessel speeds can vary considerably over a tow (Powell et al., 2003). Consequently, the relative accuracy of the straightline method and the duration/speed method is unknown. What is clear is that tow duration is a much more accurately known quantity than tow distance when only a few positions describing the tow path are known.

\section{Effort Term in CPUE and the Value of Searching Behavior}

Two questions arise during the calculation of CPUE from Captains' reports. Both involve the effort term.

The first question is the preferable use of tow duration versus tow distance to calculate effort. Tow duration provided the most accurate effort term. Tow distance was not easily estimated because of nonlinearity in tow paths, so that having only two positions to describe the towpath was inadequate, and because a single report of tow speed was likely inadequate. The correlation between catch and towduration-based CPUE was stronger than the correlation using a tow-distance-based number. More significant differences were resolved at a more significant statistical level using tow duration rather than tow distance. This reflects the lower variability in the tow duration estimates, the more consistent relationship these bore to catch, and the fact that the same boat tended to operate similarly between years with respect to this variable (Table 1, non-significant boat*year term). Finally, tow duration was not influenced by depth or latitude as main effects, although interaction terms with year were significant. Assuming the desirability of minimizing the reporting requirements onboard ship, the time of net deployment and net retrieval would be sufficient to estimate effort, whereas the position of net deployment and net retrieval would not be.

The second question concerns the decision to use or not to use searching time or searching distance as a component of effort in the calculation of CPUE. Searching is often an important component of models of fishing behavior (Gillis et al., 1995; Dorn, 2001). Further, consideration must be given to the inclusion of overnight transits as searches. Boats varied widely in the frequency of searching between tows, although the frequency never exceeded $50 \%$ of tows. Searching between tows permitted the boat to examine an area of about the length of one tow path. Searches were conducted at relatively high speed. However, searches between tows did not improve subsequent catch. Similarly, overnight transits did not improve subsequent catch even though the vessel had the luxury of searching over a much larger area. Thus, vessels, on the average, would have improved trip economics by not searching. More squid would have been landed per day and fuel use would have diminished.

Because of the apparent absence of a positive effect of searching and the lower accuracy of searching time and searching distance in comparison to tow-based 
metrics, particularly tow duration, addition of searching time or searching distance to effort did not materially improve the measurement of CPUE. Powell et al. (2003) reached the same conclusion from analysis of observed tows. In these much more closely evaluated tows, searches were similarly unsuccessful in improving catch performance. Consequently, the analyses support the use of a tow-duration-based effort term in the calculation of CPUE.

\section{Freezer Trawlers versus RSW Trawlers}

Freezer trawlers typically conduct 7 to 14-day fishing trips. RSW trawlers typically stay out for only a few days. This different approach to fishing necessitated by onboard storage requirements does not necessarily imply that the two vessels approach the task of fishing differently or reap the rewards in different measure. However, in practice, the two types of vessels were distinctive. PCA analysis showed that two variables, tow duration and tow distance, were sufficient to almost completely separate tows of these two vessel types (Fig. 12).

RSW trawlers search much less often. Shorter duration trips place a premium on tow time and, as a consequence, these captains almost never search between tows. As a consequence, tows are longer. In fact, tows are about as long as the sum of tow and search time for freezer trawlers. RSW trawlers tow at slightly lower speed. Nevertheless, RSW trawlers cover longer distances while towing. Their catch per tow is lower, although the difference between RSW trawlers and freezer trawlers is not significant, but, as a consequence of lower catch and longer duration tows, the CPUE for RSW trawlers is distinctly lower than for freezer trawlers. Managing the I. illecebrosus fishery using an industry-based reporting scheme would require information on both vessel types.

\section{Large versus Small Vessels}

Boats were assigned to two vessel size categories, relative to a $30-\mathrm{m}$ length discriminator. PCA analysis showed that two variables, tow speed and deviation from a linear path, were sufficient to almost completely separate tows of these two vessel types (Fig. 13).

Smaller vessels search much less often (Table 4). Tow durations are about the same; however, tow distances tend to be somewhat longer for the larger vessels. This is principally a function of higher tow speeds (Table 4). Smaller vessels tend to tow in a straight path. Deviations in distance between the straight-line estimate and the estimate from tow speed and tow duration were negligible for this class of vessels and averaged $16.7 \%$ for larger vessels (Table 4). Catch per tow was higher by more than a factor of two for larger vessels. As a consequence of the higher catch and longer tows, CPUE was significantly higher also for the larger vessels (Table 4). Managing the $I$. illecebrosus fishery using an industry-based reporting scheme would require information on both vessel types.

\section{Year, CPUE and Catch Trends}

We utilized the Captains' reports to evaluate trends in catch and CPUE for the fishing years 1999, 2000, and 2001. Our analyses are not corrected for the relative proportion of RSW and freezer trawlers in the fishing fleet, because we do not have information on the relative distribution of catch between these two vessel types over this period. Thus, the trends we observe in the present data set may diverge somewhat from the trends for the entire fishery.

CPUE did not vary significantly with time-of-year. Typically, I. illecebrosus moves onto the shelf edge in May/June and then back offshore in September/ October. As a consequence of this migration, one might anticipate CPUE to increase early in the search as more squid move onshore and decrease late in the season as the squid move back offshore (Basson et al., 1996). The latter immigration might look much like a reduction in abundance as a consequence of fishing (Basson et al., 1996). In 1999-2001, however, CPUE did not show any temporal pattern. The onshore migration was likely missed by the fishery, as possibly was the offshore migration. The absence of a decline in CPUE during the summer suggests that the fishery did not significantly impact the stock, a fact that is in support of analyses of fishing mortality rate by NEFSC (MS 1999) wherein the same conclusion was suggested from independent data.

Although time of year (year-day) did not influence catch to any large extent, and did not influence CPUE at all, as a main effect, the interaction of year-day with year was highly significant for both, indicating that the trends in catch and CPUE within the year varied between years. Most tow and catch variables differed significantly in value between years. Significant interaction terms between the main effects of boat, RSW versus freezer trawler, and vessel length with year were also often significant. The influence of vessel on CPUE between years, as revealed by a significant interaction between boat and year (Table 2), was not carried through when boats were divided into RSW and freezer types or length categories. Year effects seem 
to relate to individual boat performance. One cannot dismiss the possibility that the origin of the significant interaction terms lies in the different distributions of vessel reports within the year among years in this limited data set. Increased reporting coverage would be required to evaluate this possibility.

A degree of nonlinearity exists in the relationship between CPUE and catch, such that CPUE tends to increase more than catch (see also Rosenberg et al., 1990). This likely reflects a maximum haul quantity that, if taken in a short period of time, results in a higher CPUE. Thus, one might anticipate average tow time declining in years of particularly high catch. Years 1999-2001 that were the focus of this study were years when total catch was below the long-term average. As a consequence, CPUEs and tow durations may differ in high-catch years.

\section{Environmental Gradients}

One might expect I. illecebrosus catches to vary with depth and latitude since squid move onshore and offshore and migrate north and south during the year (Brodziak and Hendrickson, 1999). CPUE was little affected by these two variables. Depth consistently influenced CPUE and tow metrics more than did latitude. The interaction of year and depth was also significant as it influenced CPUE. The assumption is that squid were differentially distributed between years across depths and this influenced the location of fishing and CPUE. The strong variability between years is in keeping with the yearly variation in hydrography, particularly the relative position of the central shelf 'cold pool' and the wall of the Gulf Stream with respect to the shelf edge (Bowman, 1977). Movements of these water masses probably influence the onshelf movement of I. illecebrosus. The 1999-2001 data do not permit investigation of the depth effect on squid spatial distribution, because we do not have information on abundance outside the fished area, although the data are consistent with a general onshore-offshore movement of squid being of greater importance in determining CPUE than the alongshore component of migration. The data do suggest that a vessel-based reporting system would be less influenced by the latitudinal distribution of vessels than by the mix of vessel sizes and onboard storage methods (RSW versus freezer) providing the data.

\section{Conclusions}

The primary purpose of this study was to evaluate the factors that would influence the development of a vessel-based reporting system for real-time management of the I. illecebrosus fishery. Reporting accuracy can be assessed from independent reporting of vessel position over time. The various vessel tasks performed during the day vary in their characteristic vessel speeds, durations and distances traveled. This variation permits independent verification of the accuracy of Captains' recorded tow times and tow positions.

CPUE can be calculated in a number of different ways. Calculation of CPUE using tow duration as the effort term minimizes the influence of erroneous reporting and removes the error introduced by a few known tow positions for tows with a nonlinear towpath. CPUE calculated using duration as the error term consistently provided more information in statistical analyses. The addition of searching time in effort was not warranted. Searching time did not directionally influence catch. Thus, addition of searching time simply increased the variability in CPUE.

The data suggest that Captains' reports of the time of net deployment and retrieval, along with catch, may be adequate for most purposes, provided that the mix of vessel types reporting is carefully determined. Freezer trawlers differed significantly from RSW trawlers in CPUE. Small vessels $(<30 \mathrm{~m})$ differed significantly from large vessels $(>30 \mathrm{~m})$ in CPUE. These differences were produced by significant differences in tow duration, distance, and speed (Fig. 12, 13). Thus, the mix of boats, if not accurately reflective of the ongoing fishery, would produce a biased evaluation of fishery CPUE. On the other hand, careful attention to the coast-wide distribution of vessels seems less essential. Latitude influenced CPUE relatively little. Depth was more consequential, but one assumes that depth is a function of squid availability and would vary during the year on an individual boat basis. Nevertheless, including depth of tow in the reporting stream would seem warranted.

The suggestion was made by NEFSC (MS 1999) that the I. illecebrosus fishery catches a relatively small fraction of the available squid. That fact would limit the change in CPUE through the season produced by fishing mortality. Trends in CPUE for the 1999-2001 period support this conclusion. CPUE did not vary significantly with year-day. The onshore-offshore migration of I. illecebrosus should substantially complicate the interpretation of CPUE at the beginning and ending of the seasons. In the three years of this study, CPUE did not vary much over the fishing season, 
suggesting that a large change in abundance from migration did not occur during the fishing season in any of these three years. As the catch for all three years was below the long-term average, generalizing from these observations should be done with care.

\section{Acknowledgements}

We thank the owners and operators of the fishing vessels for taking part in this study. The study was funded by the Fisheries Information and Development Center of New Jersey. We appreciate this support.

\section{References}

AGNEW, D. J., J. R. BEDDINGTON, R. BARANOWSKI, S. DES CLERS and C. P. NOLAN. 1998. Approaches to assessing stocks of Loligo gahi around the Falklands Islands. Fish. Res., 35: 155-169

BAsSON, M., J. R. BEDDINGTON, J. A. CROMBIE, S. J. HOLDEN, L. V. PURCHASE and G. A. TINGELY. 1996. Assessment and management techniques for migratory annual squid stocks: the Illex argentinus fishery in the Southwest Atlantic as an example. Fish. Res., 28: 3-27.

BOWMAN, M. J. 1977. Hydrographic properties. MESA New York Bight Atlas Monograph 1, New York Sea Grant, Albany, New York, 78 p.

BRODZIAK, J. and L. HENDRICKSON. 1999. An analysis of environmental effects on survey catches of squids Loligo pealei and Illex illecebrosus in the Northwest Atlantic. Fish. Bull., 97: 9-24.

DAWE, E. G. and L. C. HENDRICKSON. MS 1998. A review of the biology, population dynamics, and exploitation of short-finned squid in the Northwest Atlantic
Ocean, in relation to assessment and management of the resource. NAFO SCR Doc., No. 59, Serial No. N3051, $33 \mathrm{p}$.

DORN, M.W. 2001. Fishing behavior of factory trawlers: a hierarchical model of information processing and decision-making. ICES J. Mar. Sci., 58: 238-252.

GILliS, D. M., E. K. PIKITCH and R. M. PETERMAN. 1995. Dynamic discarding decisions: Foraging theory for high-grading in a trawl fishery. Behav. Ecol., 6: 146154.

HENDRICKSON, L. C., J. BRODZIAK, M. BASSON and P. RAGO. MS 1996. Stock assessment of northern shortfin squid in the Northwest Atlantic during 1993. NEFSC Ref. Doc., No. 96-65g, 63 p.

MAFMC. 1998. Amendment 8 to the Atlantic mackerel, squid, and butterfish fishery management plan. MidAtlantic Fisheries Management Council, 295 p.

MORALES-BOJÓRQUEZ, E., S. MARTÍNEZ-AGUILAR, F. ARREGUÍN-SÁNCHEZ and M. O. NEVÁREZMARTÍNEZ. 2001. Estimates of catchability-of-length for the jumbo squid (Dosidicus gigas) fishery in the Gulf of California, Mexico. Cal COFI Rep., 42: 167171.

NEFSC. MS 1999. 29 $9^{\text {th }}$ Northeast regional stock assessment workshop (29 $9^{\text {th }}$ SAW): Stock assessment review committee (SARC) consensus summary of assessments. NEFSC Ref. Doc., No. 99-14, 347 p.

PATTERSON, K. R. 1998. Assessing fish stocks when catches are misreported: model, simulation tests, and application to cod, haddock, and whiting in the ICES area. ICES J. Mar. Sci., 55: 878-891.

POWELL, E. N., A. J. BONNER, B. MULLER and E. A. BOCHENEK. 2003. Vessel time allocation in the US Illex illecebrosus fishery. Fish. Res., 61: 35-55.

ROSENBERG, A. A., G. P. KIRKWOOD, J. CROMBIE and J. R. BEDDINGTON. 1990. The assessment of stocks of annual squid species. Fish. Res., 8: 335-350. 